\title{
Observations of the Diffraction of Evanescent X Rays at a Crystal Surface
}

\author{
P. L. Cowan and S. Brennan ${ }^{(a)}$ \\ Quantum Metrology Group, National Bureau of Standards, Gaithersburg, Maryland 20899 \\ Terrence Jach \\ Quantum Metrology Group and Surface Science Division, National Bureau of Standards, \\ Gaithersburg, Maryland 20899 \\ and \\ M. J. Bedzyk ${ }^{(\mathrm{b})}$ and G. Materlik
Hamburger Synchrotron Strahlungslabor HASYLAB at DESY, D-2000 Hamburg 52, \\ Federal Republic of Germany \\ (Received 17 January 1986)
}

\begin{abstract}
Diffraction of $\mathrm{x}$ rays from a crystal during total external reflection imposes structure on the $\mathrm{x}$-ray wave field in three dimensions. Standing-wave interference modulates the $x$-ray intensity parallel to the surface, while boundary effects damp the intensity with distance normal to the surface both within the crystal and in the region above the surface. Experiments on carefully prepared Ge single crystals show that the $x$-ray wave field can be manipulated to provide model-independent information on the structure of surfaces.
\end{abstract}

PACS numbers: $61.10 . J v, 07.85 .+n, 61.10 . \mathrm{Dp}, 68.35 . \mathrm{Bs}$

Recently a number of new techniques for structure analysis have been developed which are based on the physical optics of $x$ rays. For example, $x$-ray standingwave (XSW) methods, ${ }^{1-5}$ based on $\mathrm{x}$-ray interference, provide model-independent information on the lattice positions of impurities ${ }^{1}$ or adsorbates. ${ }^{2-5}$ Other studies ${ }^{6-9}$ exploit the shallow penetration of evanescent $\mathrm{x}$ rays to obtain selective surface sensitivity or depth profiles. We report experimental results for a novel condition, the diffraction of evanescent $x$ rays during total external reflection (DEXTER), which combines the features of XSW and evanescent wave studies. We have evidence for an additional phenomenon, superficial $x$ rays, which suggests that depth profiles can be obtained above interfaces as well as below.

The experimental arrangement was similar to previous glancing-incidence scattering experiments, ${ }^{7}$ but with several important distinctions. The sample was oriented so that a beam of monochromatic $\mathrm{x}$ rays simultaneously made a small glancing incident angle with the surface and satisfied the Bragg condition for diffraction from structure whose reciprocal-lattice vector was parallel to the surface. In contrast to the case studied by Eisenberger and co-workers, ${ }^{7}$ the Bragg diffraction arose from the bulk crystal structure itself rather than an overlayer or surface reconstruction. This was to assure significant multiple scattering, following Golovin and Imamov. ${ }^{10}$ For the first time, the specularly reflected beam was studied as well as the diffracted beam, and the incident beam divergence was strictly limited in both transverse directions in order to approximate closely predictions based on plane-wave theory. ${ }^{11-14}$ Finally, an energydispersive $\mathrm{Si}(\mathrm{Li}) \mathrm{x}$-ray detector was positioned to observe $\mathrm{x}$-ray fluorescence, as in an evanescent $\mathrm{x}$-ray absorption ${ }^{8}$ or an XSW measurement.

Measurements were performed at the RÖMO station of the Hamburger Synchrotron Strahlungslabor (HASYLAB). The monochromator was designed for XSW experiments, including diffracted-beam feedback stabilization. ${ }^{15}$ The sample chosen was a dislocation-free germanium crystal with an optically flat (1i1) surface. Final surface preparation included bromine passivation, ${ }^{2,3}$ and the sample was kept in a dry helium atmosphere during the measurements to inhibit overlayer growth. The sample's aximuthal orientation was adjusted so that (220) planes perpendicular to the surface diffracted the incident beam $(h v=8.05 \mathrm{keV})$. The sample was mounted on a goniometer which allowed independent control of the glancing incidence angle $\phi$ and the diffraction angle $\theta$.

The experimental arrangement permitted the study of the DEXTER effects by two approaches. First, reflectivity versus $\phi$ was measured for several constant values of $\theta$. In this way the effects of the varying diffraction condition on the total reflection were explored. Secondly, the diffracted flux versus $\theta$ was measured for different $\phi$ values. This demonstrated the influence of the reflection condition upon the diffraction. In both types of measurements the diffracted and reflected flux (intensity integrated over area) and the fluorescence spectra were simultaneously recorded.

When there is no diffraction involved, the specular reflectivity follows a steplike Fesnel curve. ${ }^{16}$ The critical incidence angle $\phi_{c}$, below which the reflectivity abruptly increases, is found from the mean dielectric susceptibility of the substrate. At this demarcation, the internal refracted beam changes from a homogeneous plane wave to an evanescent wave which decays exponentially within a 
few nanometers of the interface. According to recent theory, ${ }^{11,14}$ the existence of strong Bragg diffraction should change the reflectivity curve into a double step. Simply stated, the diffracted beam interferes with the refracted beam within the crystal to produce an $x$-ray standing wave. In general, this standing wave can be decomposed into a linear combination of a standing wave with nodes on the atomic planes (the $\alpha$ wave) and one with antinodes on the planes ( $\beta$ wave). These component standing waves see a localized susceptibility whose real and imaginary components are respectively smaller or larger than the mean. Hence, the reflectivity can be expected to increase with decreasing $\phi$ in two stages as first the $\beta$ wave and then the $\alpha$ wave transform from propagating waves to evanescent waves.

Figure 1 shows two examples of $\phi$ scans for constant $\theta$. When $\theta$ is near the Bragg angle $\theta_{\mathrm{B}}$, the measured reflectivity curves [Fig. 1(a)] clearly show the predicted twostep behavior. For comparison calculated curves based on Ref. 14, Eq. (5), are shown for $\theta-\theta_{\mathrm{B}}=-2$ and -24 $\mu$ rad. Note that the calculated curves have been modified to account for the beam divergence and the response envelope imposed by the finite sample size and beam cross section. The diffracted flux [Fig. 1(b)] recorded simultaneously with the reflectivity curves of Fig. 1(a)

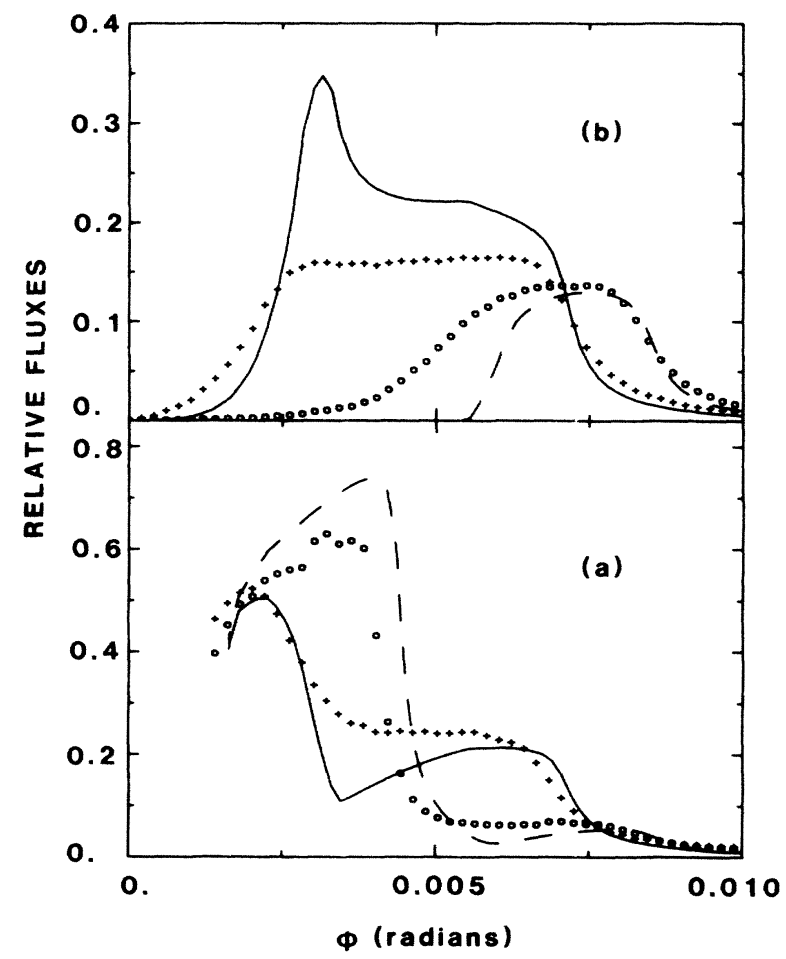

FIG. 1. Measured and calculated intensities vs $\phi$ for constant $\theta$. (a) Specular beam: experiment, $\theta-\theta_{\mathrm{B}}=-2 \mu \mathrm{rad}$ (plusses); theory, $\theta-\theta_{\mathrm{B}}=-2 \mu \mathrm{rad}$ (solid curve); experiment, $\theta-\theta_{\mathrm{B}}=-23 \mu \mathrm{rad}$ (circles); theory, $\theta-\theta_{\mathrm{B}}=-24 \mu \mathrm{rad}$ (dashed curve). (b) Diffracted beam under the same conditions. show strongest signal at $\phi$ values between the two reflectivity steps. This external diffracted beam is generated by the evanescent $\beta$ wave as shown by the qualitative agreement of the data with curves calculated from Ref. 14, Eq. (7). For $\theta-\theta_{\mathrm{B}}=-2 \mu \mathrm{rad}$ the calculated curve shows a cusp at $\phi \approx 3 \mathrm{mrad}$ which is missing in the data. The absence of the peak can be attributed to a number of experimental conditions, most likely the presence of a thin $(1-2 \mathrm{~nm})$ overlayer.

Figure 2(a) shows the dependence of the specular reflectivity on the diffraction angle $\theta$ for three values of $\phi$ near the critical angle $\phi_{c}=5.7 \mathrm{mrad}$. For $\phi<\phi_{c}$ the reflectivity dips near the Bragg condition as photons are diverted from the reflected beam to the diffracted beam. Increasing $\phi$ above the critical angle transforms the reflectivity dip at $\theta \approx \theta_{\mathrm{B}}$ into a peak. This peak is consistent with Fig. 1(a) in which the reflectivity stays high for relatively large $\phi$ when the diffraction condition is met.

Observations of the diffracted beam for the same values of $\phi$ [Fig. 2(b)] show that the flux abruptly drops to zero for $\theta<\theta_{\mathrm{B}}$. This behavior has a simple, kinematic explanation. External to the crystal, conservation of energy requires the incident $\left(\mathbf{K}_{0}\right)$ and diffracted $\left(\mathbf{K}_{H}\right)$

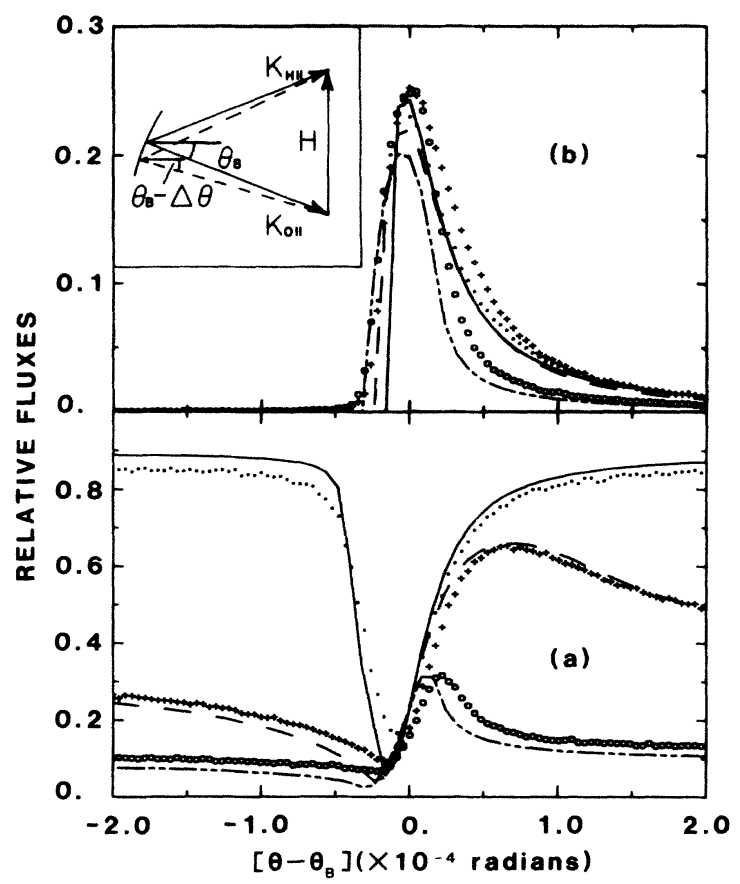

FIG. 2. Measured and calculated intensities vs $\theta-\theta_{\mathbf{B}}$ for three values of constant $\phi$. (a) Specular beam: theory, $\phi=4.7$ mrad (solid curve); experiment, $\phi=4.7 \mathrm{mrad}$ (filled circles); theory, $\phi=5.55 \mathrm{mrad}$ (dashed curve); experiment, $\phi=5.55$ mrad (plusses); theory, $\phi=6.35 \mathrm{mrad}$ (dot-dashed curve); experiment, $\phi=6.35 \mathrm{mrad}$ (open circles). (b) Diffracted beam under the same conditions. Inset: Conservation of transverse momentum. Note that as $\theta$ decreases with constant $\left|\mathbf{K}_{0 \text { III }}\right|$ (i.e., constant $\phi_{0}$ ) the magnitude of $\mathbf{K}_{H \|}$ must increase. 
beam wave vectors to have equal magnitudes. At the same time the transverse components, $\mathbf{K}_{0 \|}$ and $\mathbf{K}_{H \|}$ must differ by exactly the reciprocal lattice vector $\mathbf{H}$ [see inset to Fig. 2(b)]. As the incident-wave-vector direction changes to larger $\theta$ both conditions can be satisfied by a decrease in $\mathbf{K}_{H \|}$ and an increase in the normal component of $\mathbf{K}_{H}$. For decreasing $\boldsymbol{\theta}$ there is a limit where the transverse component of the diffracted wave vector equals its total magnitude, $K_{H \|}=K_{H}$. Beyond this limit no real value for $K_{H}$ is allowed, but a complex solution is possible. As with internal evanescent waves, ${ }^{8}$ an imaginary component of the wave vector means that the wave-field intensity will be damped with distance from the surface, but in this case the evanescent diffracted wave is external to the substrate. Thus, the diffracted-beam detector, which is in the far field, may detect no flux even though there may be significant intensity in the near field of the surface. This fact will become significant in the discussion below.

The $x$-ray standing-wave behavior for the DEXTER case was studied via observations of $x$-ray fluorescence versus $\theta$ and $\phi$. Under the experimental conditions chosen, only the Ge $L$ fluorescence from the crystal was observed. Figure 3(a) gives an example of this fluorescence signal versus $\theta$ for $\phi=4.6 \mathrm{mrad}<\phi_{c}$. The solid curve shows the calculated fluorescence for $\mathrm{Ge}$ atoms on the (220) planes going into the crystal, while the dashed curve assumes a hypothetical atomic location at intersti-

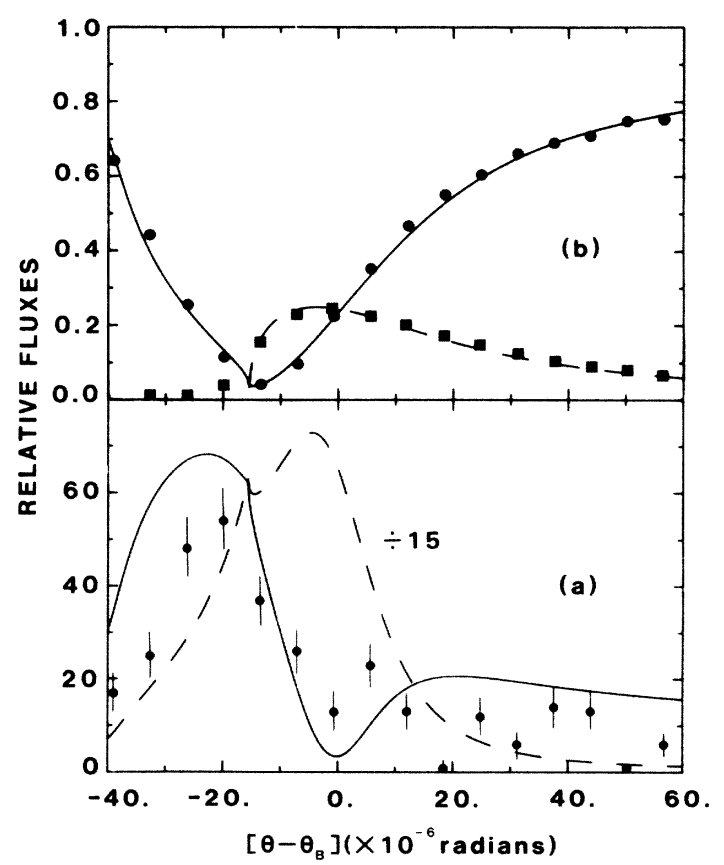

FIG. 3. (a) Ge $L$ fluorescent signal (with error bars) vs $\theta-\theta_{\mathrm{B}}$ for $\phi=4.71 \mathrm{mrad}$ (see text). (b) Specular-beam intensity (circles), experimental; solid curves, theoretical) and diffracted-beam intensity (squares, experimental; dashed curve, theoretical) vs $\theta-\theta_{\mathrm{B}}$. tial sites, between the (220) planes. The curves were normalized with use of the fluorescence observed at $\phi>\phi_{c}$, with $\theta$ far from $\theta_{\mathrm{B}}$. The difference in magnitudes of the two calculated curves is indicative of the different penetration depths of the $\alpha$ and $\beta$ waves. ${ }^{13,14}$

The observed Ge fluorescence [Fig. 3(a)] differs markedly from the calculated fluorescence curve for atoms at interstitial sites, but agrees well with the prediction for fluorescing atoms on the (220) planes. This confirms that an $\mathrm{X}$-ray standing wave was established in the DEXTER case and that the behavior of the standing wave was well charactrerized by the existing theory. ${ }^{11,14}$ The clear distinction between the theoretical curves for fluorescing atoms at different locations indicates that this $\mathrm{XSW}$ effect can be exploited to determine atomic registry, parallel to an interface, of impurities or adatoms. This has also been accomplished by previous XSW measurements, ${ }^{3,5}$ but the DEXTER case does not involve triangulation of separate XSW mesurements as does the off-normal Bragg method, ${ }^{3}$ nor must the sample be incorporated into a monolithic x-ray interferometer. ${ }^{5}$ Furthermore, in DEXTER the penetration of the $x$-ray fields can be controlled.

One can observe a curious effect in Fig. 3. The fluorescent intensity is highest for $\theta-\theta_{\mathrm{B}}=-20 \mu \mathrm{rad}$ when the observed specular and diffracted fluxes [Fig. 3 (b)] are very small. Normally, one might ascribe increasing fluroescence either to increased penetration of the $x$ rays, or to a change in the phase of an $x$-ray standing wave. The measurement was taken with constant $\phi<\phi_{c}$, so that in the absence of diffraction, the penertration should not change. Similarly, if the x-ray standing wave arises from the interference of two or more plane waves, one must ask what is interfering with the incident wave. We contend that a strong diffracted beam must be present at the crystal surface even though it did not reach the detector. This is additional evidence that the diffracted beam can become evanescent above the surface as discussed earlier. We term this effect a "superficial $\mathrm{x}$-ray wave" by analogy to the optical effect invoked by Fano to explain anomalous diffraction from gratings. ${ }^{17}$ This superficial wave is expected to decay exponentially within a few nanometers of an interface, ${ }^{14,18}$ and might be used in the same way as evanescent $x$ rays. ${ }^{6,8,9}$ For example, during the transition of the diffracted beam from plane wave to superficial wave the fluorescence from an overlayer should become restricted to the vicinity of the interface.

In conclusion, we have observed for the first time novel effects involving $\mathrm{x}$-ray reflection, diffraction, interference, and evanescence during glancing-incidence diffraction. Aside from the interest intrinsic in the physical optics of this case, several of these phenomena have potential applications for structural determination, especially for surfaces and interfaces. The x-ray standing-wave effect is particularly promising since registration is estab- 
lished directly and, unlike in previous XSW measurements, the $\mathrm{x}$-ray penetration is limited to the near surface. The capabilities of evanescent $x$ rays to determine depth profiles are extended by the DEXTER condition, especially in that the interfacial regions of overlayers may be studied with superficial $x$ rays. We have also shown that important information can be gained from the specularly reflected flux and from the $x$-ray fluorescence signal as well as the diffracted flux usually observed during glancing incidence scattering.

The authors thank Dr. K. Wieteska, from the Institute of Atomic Energy, Otwock-Swierk, Poland, for help in sample alignment at HASYLAB, and Mr. J. Fuller and Dr. A. Henins for help with sample preparation at the National Bureau of Standards.

\footnotetext{
(a) Permanent address: Stanford University, Box 4349, Bin 69, Stanford, CA 94305.

(b) Permanent address: Cornell High Energy Synchrotron Source, Cornell University, Ithaca, NY 14853.

${ }^{1}$ B. W. Batterman, Phys. Rev. Lett. 22, 703 (1969).

${ }^{2}$ P. L. Cowan, J. A. Golovchenko, and M. F. Robbins, Phys. Rev. Lett. 44, 1680 (1980).

${ }^{3}$ J. A. Golovchenko, J. R. Patel, D. R. Kaplan, P. L. Cowan, and M. J. Bedzyk, Phys. Rev. Lett. 49, 560 (1982).

${ }^{4}$ G. Materlik and J. Zegenhagen, Phys. Lett. 104A, 7 (1984).

${ }^{5}$ G. Materlik, A. Frahm, and M. J. Bedzyk, Phys. Rev. Lett. 52, 441 (1984).
}

${ }^{6}$ B. C. Lu and S. A. Rice, J. Chem. Phys. 68, 5558 (1978).

${ }^{7}$ W. C. Marra, P. Eisenberger, and A. Y. Cho, J. Appl. Phys. 50, 6927 (1979); P. Eisenberger and W. C. Marra, Phys. Rev. Lett. 46, 1081 (1981).

${ }^{8}$ R. S. Becker, J. A. Golovchenko, and J. R. Patel, Phys. Rev. Lett. 50, 153 (1983).

9J. M. Bloch, M. Sansone, F. Rondelez, D. G. Peiffer, P. Pincus, M. W. Kim, and P. M. Eisenberger, Phys. Rev. Lett. 54, 1039 (1985).

${ }^{10}$ A. L. Golovin and R. M. Imamov, Kristallografiya 29, 410 (1984) [Sov. Phys. Crystallogr. 29, 248 (1984)]; A. L. Golovin, R. M. Imanov, and S. A. Stepanov, Acta Crystallogr., Sect. A 40, 225 (1984).

${ }^{11}$ V. G. Baryshevskii, Pis'ma Zh. Tekh. Fiz. 2, 112 (1976) [Sov. Tech. Phys. Lett. 2, 43 (1976)].

${ }^{12}$ A. M. Afanas'ev and M. K. Melkonyan, Acta Crystallogr., Sect. A 39, 207 (1983).

${ }^{13}$ P. A. Aleksandrov, M. K. Melkonyan, and S. A. Stepanov, Kristallografiya 29, 376 (1984) [Sov. Phys. Crystallogr. 29, 226 (1984)].

${ }^{14}$ P. L. Cowan, Phys. Rev. B 32, 5437 (1985).

${ }^{15}$ A. Krolzig, G. Materlik, and J. Zegenhagen, Nucl. Instrum. Methods Phys. Res 208, 613 (1983); A. Krolzig, G. Materlik, M. Swars, and J. Zegenhagen, Nucl. Instrum. Methods Phys. Sect. A 219, 430 (1985).

${ }^{16} \mathrm{R}$. W. James, The Optical Principles of the Diffraction of $X$-Rays (Ox Bow Press, Woodbridge, CT, 1982).

${ }^{17}$ U. Fano, J. Opt. Soc. Am. 31, 213 (1941).

${ }^{18}$ A. V. Andreev, E. K. Kov'ev, Yu. A. Matveev, and Yu. V. Ponomarev, Pis'ma Zh. Eksp. Teor. Fiz. 35, 412 (1982) [JETP Lett. 35, 508 (1982)]. 